\title{
Estudo comparativo entre a porca convencional e a porca para fixação óssea (PFO): avaliação em laboratório*
}

\author{
Comparative study of the conventional nut and the nut \\ for bone fixation (PFO): laboratory evaluation
}

\author{
Alberto Naoki Miyazaki ${ }^{1}$, Marcelo Fregoneze², Pedro S. DoneuX³ ${ }^{3}$ Luciana Andrade Silva ${ }^{3}$, \\ Ronaldo Roncetti ${ }^{4}$, Ralph Klassen ${ }^{4}$, Maurício G. Walrigues ${ }^{4}$, Sérgio LUIZ CheCCHIA ${ }^{5}$, \\ Marcelo Tomanik MerCadante ${ }^{6}$
}

\section{RESUMO}

Objetivo: Desenvolver uma porca para fixação óssea (PFO) com o objetivo de aumentar a estabilidade da osteossíntese interna. Métodos: Foram utilizados 16 cadáveres frescos (32 extremidades pro-

* Trabalho realizado no Departamento de Ortopedia e Traumatologia da Faculdade de Ciências Médicas da Santa Casa de São Paulo, "Pavilhão Fernandinho Simonsen" - São Paulo (SP), Brasil. Diretor: Professor Doutor Osmar Avanzi.

1. Doutor, Professor Assistente do Departamento de Ortopedia e Traumatologia da Faculdade de Ciências Médicas da Santa Casa de São Paulo - São Paulo (SP), Brasil.

2. Professor Assistente do Departamento de Ortopedia e Traumatologia da Faculdade de Ciências Médicas da Santa Casa de São Paulo - São Paulo (SP), Brasil.

3. Assistente do Grupo de Cirurgia de Ombro e Cotovelo da Faculdade de Ciências Médicas da Santa Casa de São Paulo - São Paulo (SP), Brasil.

4. Médico Estagiário do Grupo de Cirurgia de Ombro e Cotovelo da Faculdade de Ciências Médicas da Santa Casa de São Paulo São Paulo (SP), Brasil.

5. Doutor, Professor Adjunto, Chefe de Clínica do Departamento de Ortopedia e Traumatologia da Faculdade de Ciências Médicas da Santa Casa de São Paulo - São Paulo (SP), Brasil.

6. Doutor, Professor Adjunto, Chefe de Clínica do Departamento de Ortopedia e Traumatologia da Faculdade de Ciências Médicas da Santa Casa de São Paulo - São Paulo (SP), Brasil.

Endereço para correspondência: Santa Casa de Misericórdia de São Paulo, Departamento de Ortopedia e Traumatologia, "Pavilhão Fernandinho Simonsen", Rua Dr. Cesário Mota Júnior, 112, Vila Buarque - 01220-020 - São Paulo (SP), Brasil. Tel./fax: () 3222-6866. E-mail: ombro@ombro.med.br. Site: www.ombro.med.br

Recebido: 10.12.07. Autor para revisão: 6.3.08. Reapresentação: 20.10.08. Aprovado: 30.10.08.

Copyright RBO2008 ximais do úmero), sendo oito masculinos e oito femininos, com média de idade de 60,56 anos. Em cada um dos 16 cadáveres, o úmero de um dos braços foi fixado com sistema de placa-parafuso-PFO e o contralateral, com o sistema placa-parafuso-porca de metal convencional. Foi aplicada uma força sobre os sistemas, medida por meio de torquímetro, até que um dos elementos falhasse (porca de polietileno, porca convencional ou osso). Depois, foi medida a força usada individualmente por 20 ortopedistas para apertar parafusos até que acreditassem que o sistema podia ser considerado como estável. Todos os resultados foram anotados e avaliados estatisticamente. Resultados: Na avaliação da força média utilizada para aperto de parafusos, nas fixações rotineiras, individualmente, por 20 ortopedistas experientes, registrou-se a força média de $3,18 \mathrm{~N}$.m, força esta inferior à resistência do sistema placa-parafuso-PFO, que foi de $6,37 \mathrm{~N} . \mathrm{m}$. A falha do sistema no osso com a PFO ocorreu em três casos e do sistema no osso com a porca de metal, em 10. Conclusão: A porca PFO mostrou-se menos agressiva ao osso que a porca metálica convencional, apresentando menor índice de fratura no osso. A força média aplicada pelo ortopedista não é capaz de quebrar a porca e levar a falha do sistema.

Descritores - Osteoporose/lesões; Fraturas do ombro/cirurgia; Dispositivos de fixação ortopédica; $\mathrm{Ca}$ dáver 


\section{ABSTRACT}

Objective: To develop a bone fixation nut (PFO) for the purpose of increasing the internal osteosynthesis stability. Methods: 16 fresh cadavers were used (32 proximal humeral extremities), eight male and eight female, mean age of 60.56 years. In each of the 16 cadavers, the humerus of one of the arms was fixated with a PFO-plate-screw system, and the contralateral was fixated with the plate-screw-nut system made of conventional metal. A load was applied on the system and measured by a torquemeter till one of the elements failed (polyethylene nut, conventional nut, or bone). The load individually used by 20 orthopedists to tighten the screws till the point they believed that the system was stable was measured. All of the results were noted down and statistically evaluated. Results: In the evaluation of the mean load used to tighten the screws, in routine fixations, individually, by 20 experienced orthopedists, a mean load of $3.18 \mathrm{~N} . \mathrm{m}$ was found, this load being lower than the strength of the PFO-plate-screw system, that was 6.37 N.m. Failure of the system in the bone with the PFO occurred in three cases and failure of the system with the metal nut occurred in 10 cases. Conclusion: The PFO nut showed to be less aggressive to the bone than the conventional metal nut, and presented a lower incidence of bone fracture. The mean load applied by the orthopedist is not sufficient to break the nut and lead to a system failure.

Keywords - Osteoporosis/injuries; Shoulder fractures/ surgery; Orthopedic fixation devices; Cadaver

\section{INTRODUÇÃO}

O sucesso do tratamento de fraturas, deformidades e pseudartroses depende, entre outros fatores, da fixação interna estável, quando esta for a técnica utilizada. Para que a estabilidade da fratura ou pseudartrose seja alcançada, é necessário que a pressão gerada pelo parafuso seja de magnitude suficiente para produzir um contato adequado entre a placa e o osso ${ }^{(1)}$. Esta pressão muitas vezes não é obtida com os implantes convencionais de osteossíntese (placas e parafusos), principalmente na região metafisária em pacientes idosos, devido à fragilidade óssea. Exemplos destas situações são as fraturas e pseudartroses da região metafisária do terço proximal do úmero.

Vários métodos têm sido propostos para a fixação das fraturas desviadas do terço proximal do úmero, como: fixação externa ${ }^{(2)}$, redução fechada e fixação percutânea $^{(3)}$, redução aberta e fixação com fios ${ }^{(4-9)}$, e diversas técnicas de redução aberta e fixação interna com implantes metálicos e/ou amarrilhos ${ }^{(10-12)}$. Nos últimos cinco anos o uso das chamadas placas bloqueadas se tornou mais comum. Estas placas foram desenvolvidas inicialmente com o objetivo de melhorar o resultado dos casos de fraturas graves, periarticulares, de alta energia. Com a crescente dificuldade para atingir e manter a "pega" dos parafusos em síntese em regiões metafisários e/ou em osso osteoporótico, estas placas ganharam grande espaço ${ }^{(13)}$.

Inúmeros trabalhos foram realizados em laboratório demonstrando a superioridade da fixação com placas bloqueadas em relação às placas convencionais e a outros métodos de fixação, como as hastes para extremidade proximal do úmero ${ }^{(14-16)}$.

No Departamento de Ortopedia e Traumatologia da Santa Casa de São Paulo, durante muitos anos, devido ao alto custo deste tipo de material, desenvolvemos uma porca, para fixação óssea (PFO), de baixo custo e de fácil utilização, para ser usada com parafusos corticais de $4,5 \mathrm{~mm}$ com o objetivo de aumentar a estabilidade da osteossíntese interna, em situações em que a qualidade óssea fosse precária.

Self et al publicaram estudo usando porcas para a fixação de fraturas complexas da extremidade distal do úmero com colocação de placas em posição lateral e medial ${ }^{(17)}$. Kolodziej et al publicaram outro estudo biomecânico avaliando uma porca a ser usada na cortical junto à placa ${ }^{(1)}$. Embora com princípios biomecânicos diversos, ambos os estudos mostraram melhora na fixação óssea.

Testamos o comportamento e a resistência da porca PFO em ombros de cadáveres recentes, comparandoos com os da porca convencional usada pelo grupo $\mathrm{AO}^{(*)}$.

(*) Schneider E. AO Research Institute. Comunicado pessoal. Davos, Suiça. Março de 1999. 
O objetivo deste trabalho é mostrar os resultados obtidos, definindo, assim, parâmetros para seu uso na prática diária.

\section{MÉTODOS}

O trabalho foi realizado pelos Grupos de Ombro e de Trauma do Departamento de Ortopedia e Traumatologia, Pavilhão "Fernandinho Simonsen", da Santa Casa de Misericórdia de São Paulo.

A porca desenvolvida é formada por dois componentes. Uma peça metálica central onde há a rosca e outra de polietileno (RCM-1000 - ultrapeso molecular) que envolve a porção central (figura 1). A peça de polietileno é côncava e se adapta à cortical oposta do osso.
A via de acesso cirúrgica utilizada foi a deltopeitoral. O ponto de fixação da porca foi no local da inserção da borda proximal do tendão do músculo peitoral maior. No caso da PFO, a placa foi colocada lateralmente e a porca, medialmente. O parafuso foi adaptado a uma placa de compressão dinâmica na cortical lateral e a porca fixada na cortical oposta por meio de uma pinça especialmente desenvolvida para esse fim, que tem um formato anatômico que favorece sua colocação na face medial do úmero sem a necessidade de grande desperiostização (figuras 2 e 3 ). A pinça apresenta em sua extremidade de preensão um prendedor que se encaixa na ranhura existente na PFO (figuras $4 \mathrm{e}$ 5). Na mesma posição do úmero contralateral, um sis-
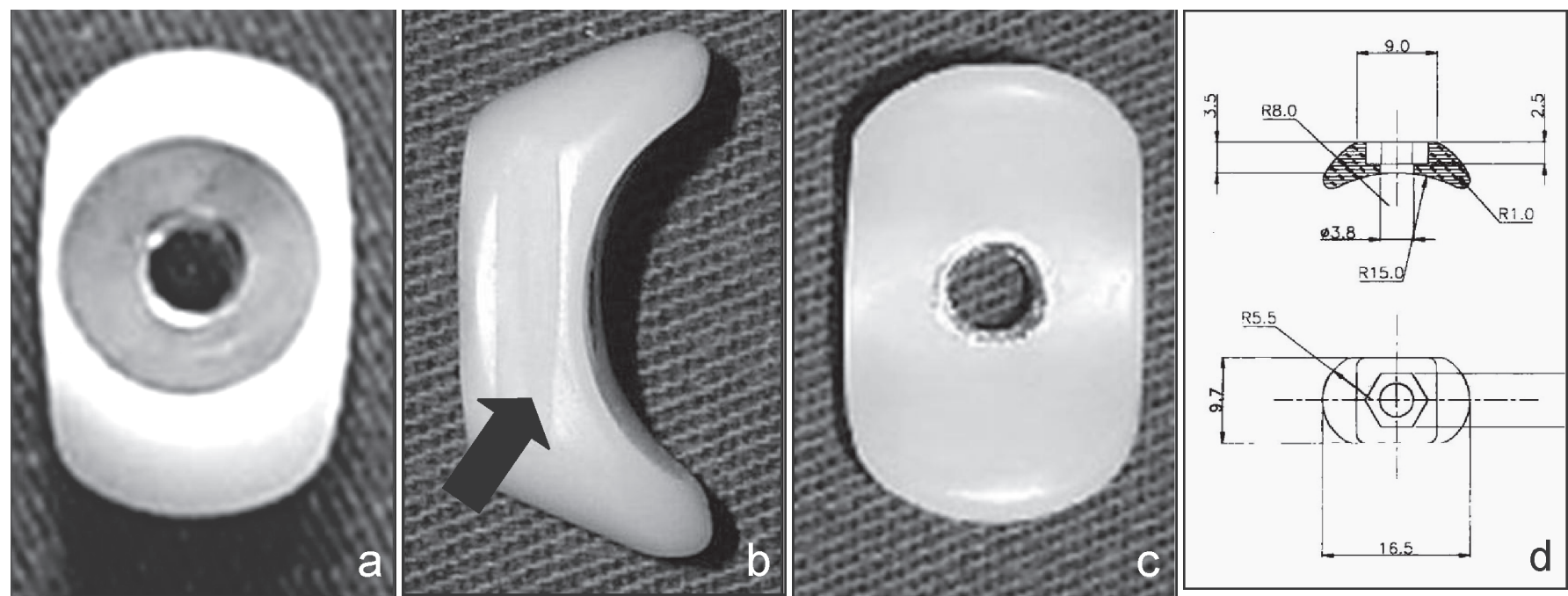

Figura 1 - PFO: (a) visão superior mostrando corpo central de metal em molde de polietileno; (b) visão lateral mostrando a forma côncava do polietileno (seta: ranhura para a encaixe da pinça); (c) visão inferior; (d) esquema com as medidas.

A superfície dela é de $206,6 \mathrm{~mm}^{2}$ (figura 1). A deformação do polietileno ocorre de forma progressiva à medida que a porca é comprimida contra o osso, até que fique perfeitamente adaptada. O momento em que a porca começa a sofrer deformação foi mensurado por um torquímetro, que mede a força em newton.metro (N.m).

Foram feitas 32 dissecções cirúrgicas em ombros de 16 cadáveres: oito eram do sexo feminino e oito do masculino, com média de idade de 60,56 anos, variando entre 44 e 75; nove eram melanodérmicos e sete, brancos (tabela 1).
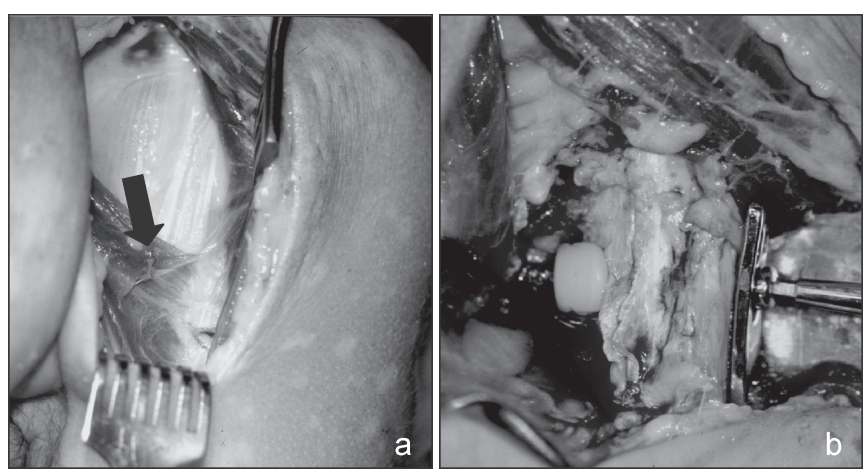

Figura 2 - Via de acesso deltopeitoral, em cadáver, (a) mostrando o local (seta) da inserção do tendão do músculo peitoral maior e (b) o sistema placa-parafuso-PFO adaptada ao osso. 
TABELA 1

Casos estudados para avaliação e comparação dos sistemas parafuso/PFO/osso e parafuso porca de metal/osso, indicando onde houve e com que força (newton.metro) ocorreu a falha

\begin{tabular}{|c|c|c|c|c|c|c|c|}
\hline Casos & Idade & Sexo & Raça & Porcas & Falha da porca & Falha do parafuso & Fratura do osso \\
\hline 1 & 71 & $\mathrm{~F}$ & $P$ & $\begin{array}{c}\text { polietileno } \\
\text { metal }\end{array}$ & $\begin{array}{c}5,5 \\
-\end{array}$ & - & $\overline{8}, 0$ \\
\hline 2 & 75 & $\mathrm{~F}$ & $P$ & $\begin{array}{c}\text { polietileno } \\
\text { metal }\end{array}$ & $\begin{array}{l}7,0 \\
-\end{array}$ & $\begin{array}{l}- \\
-\end{array}$ & - \\
\hline 3 & 65 & $\mathrm{~F}$ & $\mathrm{P}$ & $\begin{array}{c}\text { polietileno } \\
\text { metal }\end{array}$ & $\begin{array}{l}- \\
-\end{array}$ & $\begin{array}{l}- \\
-\end{array}$ & $\begin{array}{l}7,0 \\
4,5\end{array}$ \\
\hline 4 & 54 & M & B & $\begin{array}{l}\text { polietileno } \\
\text { metal }\end{array}$ & $\begin{array}{c}7,0 \\
-\end{array}$ & $\begin{array}{l}- \\
-\end{array}$ & $\begin{array}{c}- \\
7,0\end{array}$ \\
\hline 5 & 54 & $\mathrm{~F}$ & B & $\begin{array}{l}\text { polietileno } \\
\text { metal }\end{array}$ & $\begin{array}{c}4,0 \\
-\end{array}$ & 8,0 & $\begin{array}{l}- \\
-\end{array}$ \\
\hline 6 & 57 & $\mathrm{~F}$ & $\mathrm{P}$ & $\begin{array}{l}\text { polietileno } \\
\text { metal }\end{array}$ & $\begin{array}{c}6,0 \\
-\end{array}$ & 8,0 & $\begin{array}{l}- \\
-\end{array}$ \\
\hline 7 & 69 & M & B & $\begin{array}{c}\text { polietileno } \\
\text { metal }\end{array}$ & $\begin{array}{l}7,0 \\
5,0\end{array}$ & - & $\begin{array}{l}- \\
-\end{array}$ \\
\hline 8 & 65 & $\mathrm{~F}$ & $P$ & $\begin{array}{c}\text { polietileno } \\
\text { metal }\end{array}$ & $\begin{array}{l}- \\
-\end{array}$ & - & $\begin{array}{l}5,0 \\
4,5\end{array}$ \\
\hline 9 & 45 & M & B & $\begin{array}{c}\text { polietileno } \\
\text { metal }\end{array}$ & $\begin{array}{l}- \\
-\end{array}$ & $\begin{array}{l}- \\
-\end{array}$ & $\begin{array}{l}6,5 \\
6,0\end{array}$ \\
\hline 10 & 65 & M & B & $\begin{array}{l}\text { polietileno } \\
\text { metal }\end{array}$ & $\begin{array}{l}- \\
-\end{array}$ & $\begin{array}{l}6,0 \\
6,0\end{array}$ & $\begin{array}{l}- \\
-\end{array}$ \\
\hline 11 & 58 & $\mathrm{~F}$ & $P$ & $\begin{array}{c}\text { polietileno } \\
\text { metal }\end{array}$ & $\begin{array}{c}6,0 \\
-\end{array}$ & $\begin{array}{l}- \\
-\end{array}$ & - \\
\hline 12 & 56 & M & $P$ & $\begin{array}{c}\text { polietileno } \\
\text { metal }\end{array}$ & $\begin{array}{l}7,0 \\
-\end{array}$ & $\begin{array}{c}- \\
7,0\end{array}$ & $\begin{array}{l}- \\
-\end{array}$ \\
\hline 13 & 71 & M & $P$ & $\begin{array}{c}\text { polietileno } \\
\text { metal }\end{array}$ & $\begin{array}{c}6,5 \\
-\end{array}$ & - & $\overline{-}$ \\
\hline 14 & 59 & M & B & $\begin{array}{c}\text { polietileno } \\
\text { metal }\end{array}$ & $\begin{array}{c}6,5 \\
-\end{array}$ & $\begin{array}{l}- \\
-\end{array}$ & $\begin{array}{c}- \\
6,0\end{array}$ \\
\hline 15 & 61 & M & B & $\begin{array}{c}\text { polietileno } \\
\text { metal }\end{array}$ & $\begin{array}{l}7,0 \\
-\end{array}$ & $\begin{array}{l}- \\
-\end{array}$ & - \\
\hline 16 & 44 & $\mathrm{~F}$ & $P$ & $\begin{array}{l}\text { Polietileno } \\
\text { metal }\end{array}$ & $\begin{array}{c}8,0 \\
-\end{array}$ & $\begin{array}{c}- \\
7,0\end{array}$ & $\begin{array}{l}- \\
-\end{array}$ \\
\hline
\end{tabular}

$\mathrm{F}=$ feminino, $\mathrm{M}=$ masculino, $\mathrm{N}=$ negra, $\mathrm{B}=$ branca

tema semelhante foi montado utilizando-se uma porca convencional (figura 6).

Para avaliar a força que era aplicada no sistema parafuso-placa-osso PFO ou porca metálica, utilizou-se o mesmo torquímetro. Foi considerada falência do sistema quando um dos componentes não suportou a força aplicada, isto é, quando houve fratura do osso pela porca, danos no local de encaixe da chave sextavada no parafuso, quebra do parafuso ou falência da porca.
Para demonstrar o quanto de força se faz normalmente no aperto de parafusos, realizou-se uma avaliação com 20 ortopedistas experientes, membros dos diversos Grupos do Departamento de Ortopedia e Traumatologia do Pavilhão "Fernandinho Simonsen". Foi montado um sistema tradicional de placa de compressão dinâmica e três parafusos em uma diáfise de úmero; solicitou-se aos mesmos que apertassem os parafusos com a graduação de força que normalmente fa- 


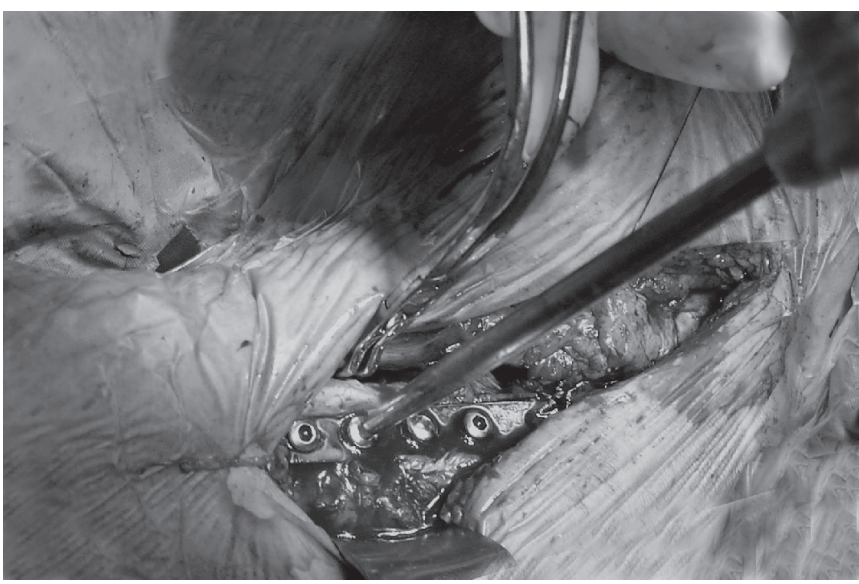

Figura 3 - Detalhe da cirurgia mostrando o aperto do parafuso e a pinça apoiada na cortical óssea oposta
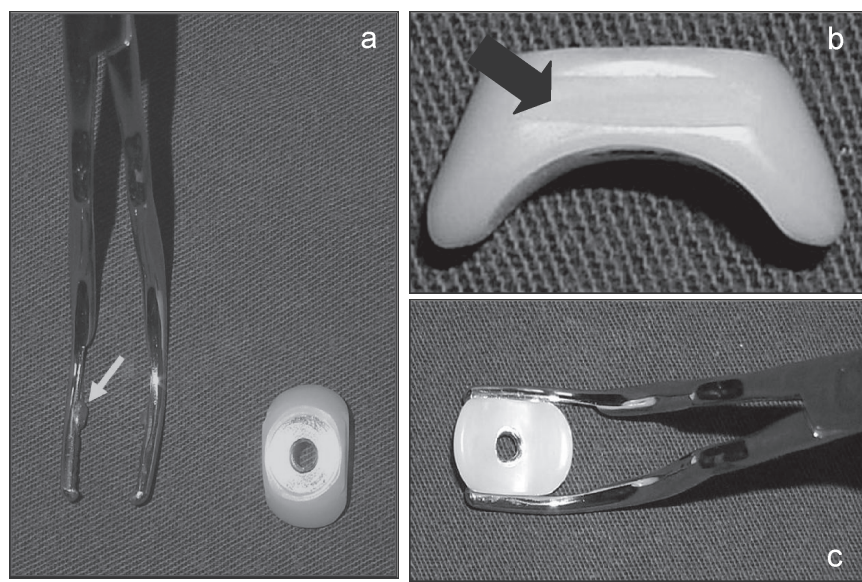

Figura 5 - Detalhe da ponta de pinça mostrando (a) "dentes" que se adaptam (b) à ranhura da PFO, e garantem (c) firmeza e precisão no posicionamento da porca na cortical óssea oposta.

zem durante o ato operatório, até o ponto em que achassem satisfatório; esta força também foi mensurada pelo torquímetro utilizado nos testes anteriores.

Os resultados obtidos nos dois sistemas - parafuso/ PFO e parafuso/porca de metal - foram avaliados estatisticamente e comparados com a média de força obtida pelos ortopedistas (teste t). Também, foram estatisticamente avaliados, pelo teste exato de Fisher, os dois sistemas em relação à falha no osso. Quando o valor de $\mathrm{p}$ foi $\leq 0,05$, os resultados foram considerados significantes.

\section{RESULTADOS}

Primeiro observamos que o componente da porca PFO de polietileno iniciou sua deformação com 2 N.m.
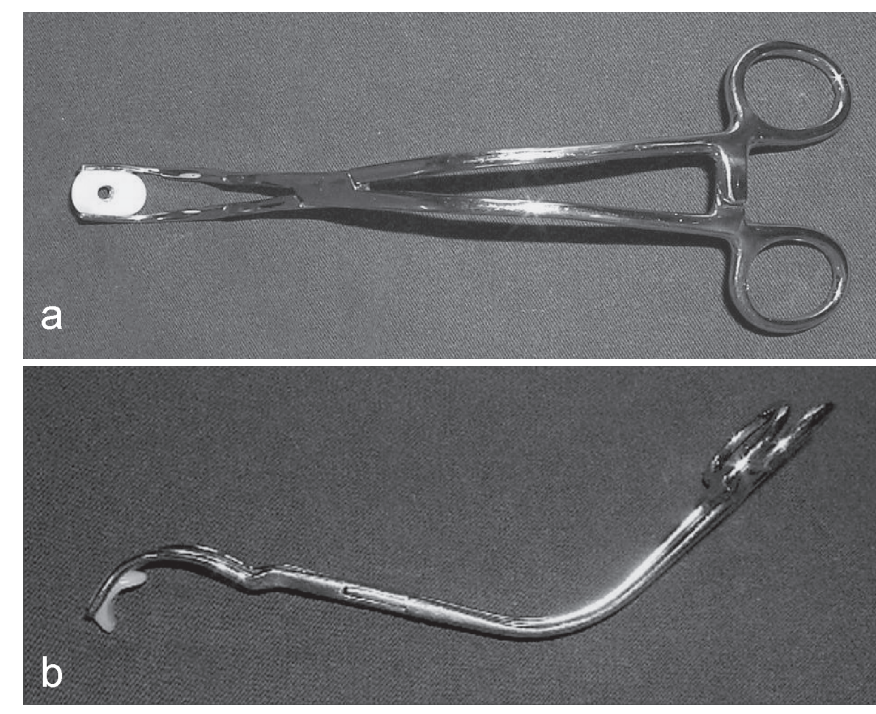

Figura 4 - Pinça para segurar a PFO na cortical óssea oposta, desenvolvida para evitar a desperiostização: (a) de frente e (b) de perfil.

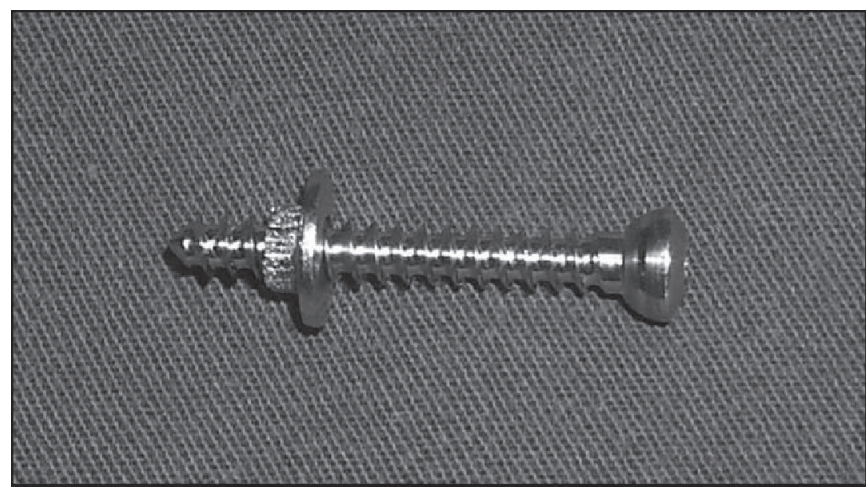

Figura 6 - Porca convencional de metal

Os sistemas falharam na porca em 13 casos, sendo que em 12 a falha ocorreu no polietileno após a aplicação da força média de $6,56 \mathrm{~N} . \mathrm{m}$. e, em um na porca de metal com força de 5,0N.m. A falha foi no parafuso em seis casos após a aplicação da força média de 7,0N.m (tabela 1).

$\mathrm{Na}$ análise estatística verificou-se que, quando comparado o sistema parafuso/PFO com o sistema parafuso/porca de metal, não houve diferença estatisticamente significativa entre ambos. Os sistemas falharam, em média, quando aplicada uma força de 6,37N.m e 6,16N.m, respectivamente (tabela 2).

Avaliando a falha do sistema em relação ao osso (fratura do osso pela porca), registramos 13 casos, sendo que em 10 casos a falha ocorreu quando foi utilizada a 
TABELA 2

\begin{tabular}{|c|c|c|}
\hline \multicolumn{3}{|c|}{$\begin{array}{l}\text { Análise estatística comparando o sistema } \\
\text { parafuso / PFO com o sistema parafuso/porca } \\
\text { de metal em relação à intensidade de força } \\
\text { aplicada durante os testes laboratoriais }\end{array}$} \\
\hline Casos & $\begin{array}{l}\text { Parafuso / PFO } \\
\text { (newton.metro) }\end{array}$ & $\begin{array}{l}\text { Parafuso/porca de metal } \\
\text { (newton.metro) }\end{array}$ \\
\hline 1 & 5,5 & 8,0 \\
\hline 2 & 7,0 & 6,0 \\
\hline 3 & 7,0 & 4,5 \\
\hline 4 & 7,0 & 7,0 \\
\hline 5 & 4,0 & 8,0 \\
\hline 6 & 6,0 & 8,0 \\
\hline 7 & 7,0 & 5,0 \\
\hline 8 & 5,0 & 4,5 \\
\hline 9 & 6,5 & 6,0 \\
\hline 10 & 6,0 & 6,0 \\
\hline 11 & 6,0 & 5,5 \\
\hline 12 & 7,0 & 7,0 \\
\hline 13 & 6,5 & 6,0 \\
\hline 14 & 6,5 & 6,0 \\
\hline 15 & 7,0 & 4,0 \\
\hline 16 & 8,0 & 7,0 \\
\hline média & 6,375 & 6,156 \\
\hline desvio padrão & 0,957 & 1,276 \\
\hline teste $\mathrm{t}$ & 0,631 & \\
\hline
\end{tabular}

porca de metal com uma força média de 5,75N.m e somente em três quando utilizada a PFO $(6,17 \mathrm{~N} . \mathrm{m})$. Comparando os mesmos sistemas por meio de análise estatística, verificamos diferença significativa $(\mathrm{p} \leq 0,05$ - teste exato de Fisher); portanto, podemos afirmar que a ocorrência de fratura no osso é mais provável no sistema porca-metal do que na PFO (tabela 1).

A média da força aplicada pelo cirurgião ortopedista no aperto do parafuso foi de $3,18 \mathrm{~N}$.m $(2,5$ a 4,25 N.m). Quando comparada com o sistema da PFO (em que a falha ocorreu em média com 6,375N.m), observamos que este último é muito mais resistente (p $<0,05$ - teste $t$ ) (tabela 3).

\section{DISCUSSÃO}

A fixação de fraturas e pseudartroses na região metafisária dos ossos longos, bem como em pacientes com osteopenia, é, muitas vezes, um desafio para o ortopedista. Tomamos como exemplo desta situação a região
TABELA 3

Força em newton.metro, aplicada pelos ortopedistas ao apertarem três parafusos, em ossos de cadáveres

\begin{tabular}{|c|c|c|c|c|}
\hline Ortopedista & Parafuso 1 & Parafuso 2 & Parafuso 3 & Média \\
\hline 1 & 2,50 & 2,50 & 2,50 & 2,50 \\
\hline 2 & 3,75 & 2,75 & 2,23 & 2,91 \\
\hline 3 & 3,75 & 4,25 & 3,00 & 3,67 \\
\hline 4 & 3,50 & 3,75 & 3,50 & 3,58 \\
\hline 5 & 1,50 & 1,50 & 2,00 & 1,67 \\
\hline 6 & 1,50 & 2,50 & 2,50 & 2,17 \\
\hline 7 & 2,50 & 3,75 & 2,00 & 2,75 \\
\hline 8 & 3,75 & 3,00 & 2,00 & 2,92 \\
\hline 9 & 3,00 & 3,75 & 3,00 & 3,25 \\
\hline 10 & 3,00 & 3,00 & 3,00 & 3,00 \\
\hline 11 & 3,75 & 3,75 & 3,75 & 3,75 \\
\hline 12 & 3,00 & 3,00 & 3,00 & 3,00 \\
\hline 13 & 4,25 & 4,00 & 4,00 & 4,08 \\
\hline 14 & 3,00 & 2,50 & 2,50 & 2,67 \\
\hline 15 & 4,25 & 4,00 & 4,00 & 4,08 \\
\hline 16 & 3,75 & 4,00 & 3,50 & 3,75 \\
\hline 17 & 3,00 & 3,00 & 2,50 & 2,83 \\
\hline 18 & 4,00 & 3,75 & 4,00 & 3,92 \\
\hline 19 & 2,50 & 4,00 & 4,25 & 3,58 \\
\hline \multirow[t]{5}{*}{20} & 3,75 & 3,00 & 3,75 & 3,50 \\
\hline & & & média & 3,18 \\
\hline & & & desvio padrão & 0,65 \\
\hline & & & limite superior & 4,45 \\
\hline & & & limite inferior & 1,90 \\
\hline
\end{tabular}

metafisária do terço proximal do úmero, cujas fraturas, principalmente em idosos, muitas vezes não podem ser estabilizadas com material de osteossíntese interna convencional (placas e parafusos), pois os parafusos simplesmente não "dão pega" (18).

Relatos recentes de vários autores demonstram os maus resultados obtidos nas osteossínteses com placas nas fraturas em três e quatro partes do terço proximal do úmero, sendo a perda da fixação da placa, com afrouxamento dos parafusos, uma das complicações ${ }^{(18-}$ 19).

A utilização de placas bloqueadas tem ajudado os cirurgiões nos casos de fraturas muito cominutas, na região metafisária ou nos casos de fragilidade óssea. No entanto, o custo elevado deste tipo de material é considerado fator limitante para seu uso. Além disso, trabalhos têm demonstrado complicações com este tipo de síntese, principalmente quando utilizado em fraturas em duas partes com traço simples, que evoluem 

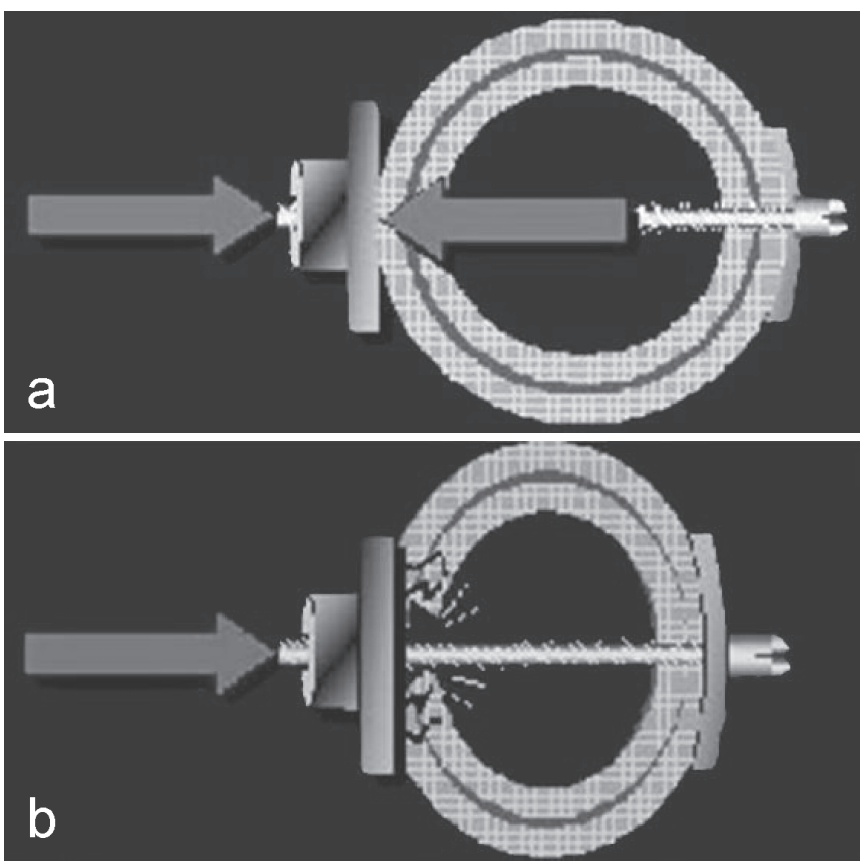

Figura 7 - Esquema da porca convencional de metal (a) mostrando contacto linear com o osso e (b) a fratura que pode advir da hiperpressão.

para pseudartrose ou em ossos muito osteoporóticos, eventualidade em que os parafusos podem penetrar na glenóide porque a cabeça do úmero "afunda" progressivamente sobre os parafusos proximais da placa $^{(13,20)}$.

Temos usado uma placa angulada de baixo perfil (placa "PFS" - Pavilhão Fernandinho Simonsen), desenvolvida na própria instituição, para fixar as fraturas em duas partes do colo cirúrgico do úmero ${ }^{(21)}$. Observamos boa fixação do fragmento proximal, porém, em alguns casos, os parafusos do fragmento distal apresentavam sinais de soltura. Este fato levou-nos a buscar uma alternativa viável, tanto do ponto de vista econômico, quanto de aplicação prática que melhorasse a fixação, principalmente nos casos de osteopenia.

Há no arsenal ortopédico uma porca convencional usada pelo grupo $\mathrm{AO}^{(10)}$. A porca "AO" deveria ser usada com parafusos de $4,5 \mathrm{~mm}$, posicionada na cortical oposta à placa, dando assim uma fixação rígida ao parafuso. $\mathrm{Na}$ realidade, ela apresentava um contato linear junto ao osso ${ }^{(1)}$, sendo abandonada, principalmente, devido a complicações, como fraturas na cortical em que era aplicada ${ }^{(*)}$.
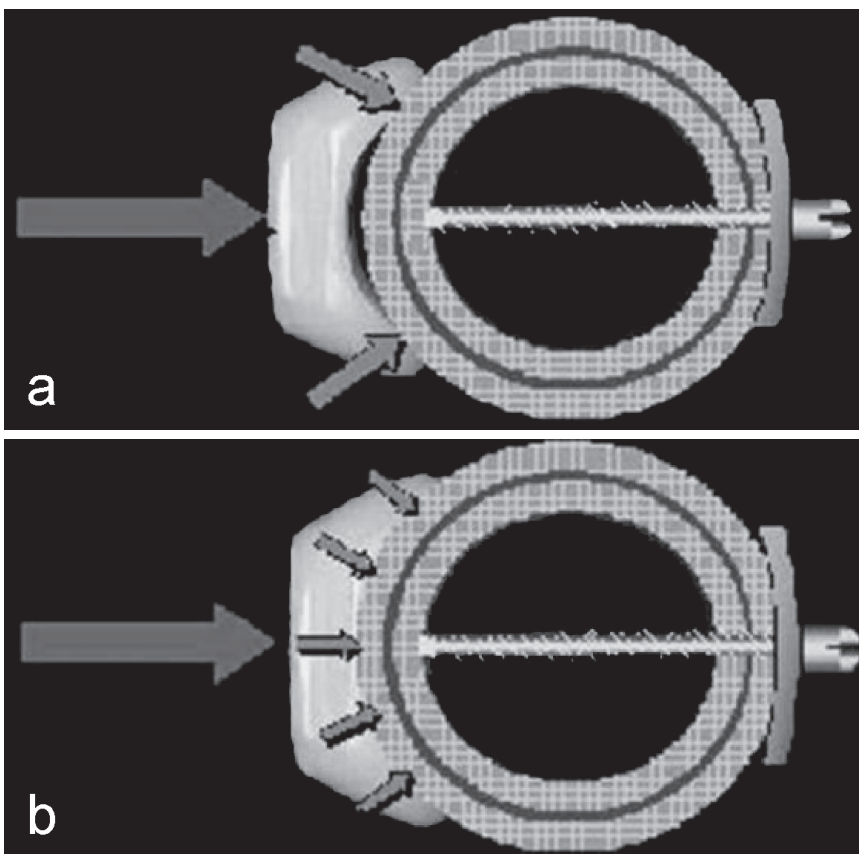

Figura 8 - Esquema da PFO (a) mostrando a porca antes de adaptação à cortical óssea e (b) adaptada à cortical óssea, diminuindo a pressão por aumento da área de contacto.

A porca PFO possui uma concavidade de polietileno que se adapta à cortical oposta do osso. O polietileno tem a função de aumentar a área de contato entre a porca e o osso, que passa de linear, como ocorre na porca de metal, onde o apoio se faz em um único ponto (figura 7), para uma superfície de $206,6 \mathrm{~mm}^{2}$ como se verifica na PFO (figura 8). Sabendo que a pressão é inversamente proporcional à superfície (pressão = massa/área), com o uso deste novo modelo de porca alcançou-se menor pressão na interface com o osso. Portanto, com essa adaptação diminuímos o risco de a porca fraturar o osso quando aplicada com determinada força que ultrapasse sua resistência.

Isso foi observado pela avaliação do comportamento biomecânico dos sistemas montados nos cadáveres. Como os dois sistemas (PFO e porca convencional) foram usados no mesmo cadáver, um lado serviu de controle para o outro. Observamos, como era esperado, que houve perfeita adaptação da concavidade do polietileno à convexidade da cortical óssea. O componente de polietileno da porca PFO deformou-se à medida que era comprimido contra o osso, até que ficou 
perfeitamente adaptado. Esta deformação iniciou-se com uma força de 2 N.m. Com a aplicação de uma força (torque) cada vez maior ao parafuso, notamos que ocorria fratura do osso em número maior de casos quando utilizada a porca convencional (três casos no lado em que foi usada a PFO e em 10 no lado em que foi utilizada a outra porca) (tabela 1). Comparando os sistemas, identificamos que as diferenças de resultado encontradas foram estatisticamente significativas ( $\mathrm{p} \leq$ 0,05 - teste $t$ ), confirmando nossa hipótese de que o aumento da área de contato obtida pelo polietileno diminui a pressão sobre o osso e, em decorrência, diminui o risco de fratura no osso cortical, praticamente eliminando a principal complicação descrita na literatura que trata de porcas convencionais.

Outro dado importante no funcionamento da PFO é a resistência máxima ao estresse antes que ocorra a quebra do polietileno e, portanto, a falha do sistema. Este valor foi de 6,37N.m, em média (tabela 2).

Precisávamos saber se um ortopedista, quando aperta um parafuso, aplica força igual ou superior à resistência da porca, pois isso levaria a falha do sistema. Para

\section{REFERÊNCIAS}

1. Kolodziej P, Lee FS, Patel A, Kassab SS, Shen KL, Yang KH, Mast JW. Biomechanical evaluation of the Schuhli nut. Clin Orthop Relat Res. 1998;(347):79-85.

2. Kristiansen B. Treatment of displaced fractures of the proximal humerus: transcutaneous reduction and Hoffmann's external fixation. Injury. 1989;20(4):195-9.

3. Hoffmann R, Khodadadyan C, Raschke M, Melcher I, Maitino PD, Haas NP. Retrograde intramedullary nailing in proximal fracture of the humerus in the elderly patient. Results of a minimally invasive management concept. Zentralbl Chir. 1998; 123(11):1232-8.

4. Kapandji A. L'ostéosynthèse par la technique des broches "en palmiers" des fractures du col chirurgical de 1'humérus. Ann Chir Main. 1989;8:39-52.

5. Resch H, Beck E, Bayley I. Reconstruction of the valgusimpacted humeral head fracture. J Shoulder Elbow Surg. 1995; 4(2):73-80.

6. Checchia SL, Miranda DL, Carneiro MU, Cassani R. Tratamento das fraturas do colo cirúrgico do úmero pela técnica de Kapandji. Rev Bras Ortop. 1993;28(1/2):43-9.

7. Ferreira Neto AA, Ferreira Filho AA, Zoppi Filho A, Benegas E, Negri JH, Machado LF, Oliveira FR. Osteossíntese das fraturas em duas e três partes da extremidade proximal do úmero tal, realizamos outro experimento, para identificar a força que um cirurgião ortopedista aplica à chave, e que é transmitida ao parafuso. Esta força variou de 2,5 a 4,25N.m, com média de 3,18N.m, isto é, inferior aos 6,37N.m necessários para que ocorra quebra do material ( $\mathrm{p} \leq 0,05)$. Com esses dados, concluímos que um ortopedista não deverá quebrar a PFO, aplicando a força transmitida por uma chave sextavada.

Há a necessidade da aplicação clínica do sistema PFO para que estes resultados encontrados em modelos biomecânicos possam ser confirmados na prática diária.

\section{CONCLUSÃO}

A ocorrência de fraturas no osso é mais provável com o uso da porca convencional do que com a porca para fixação óssea (PFO), como comprovado estatisticamente.

A força normal que um ortopedista habitualmente aplica ao parafuso através de uma chave sextavada convencional é inferior a resistência do sistema PFO.

com hastes de Ender modificadas associadas com amarrilhos de "Ethibond". Rev Bras Ortop. 1997;32(9):707-12.

8. Menniti EL, Brasil Filho R, Filardi Filho CS, Baptista MV, Daher SS. Banda de tensão intramedular com parafusos em fraturas do colo do úmero em duas e três partes: "sistema pára-quedas". Avaliação de nova técnica. Rev Bras Ortop. 1997;32(9):713-7.

9. Cuomo F, Flatow EL, Maday MG, Miller SR, McIlveen SM, Bigliani LU. Open reduction and internal fixation of two and three-part displaced surgical neck fratures of the proximal humerus. J Shoulder Elbow Surg. 1992;1:287-94.

10. Rüedi T, Schweiberer L. Escápula, clavícula, úmero. In: Müller ME, Allgöwer M, Schneider R, Willenegger, H. Manual de osteossíntese: técnicas recomendadas pelos grupos AO-ASIF. 3a ed. São Paulo: Manole; 1997.

11. Neer CS. Cirurgia do ombro. Rio de Janeiro: Revinter; 1995. p. 342-96.

12. Koukakis A, Apostolou CD, Taneja T, Korres DS, Amini A. Fixation of proximal humerus fractures using the PHILOS: early experience. Clin Orthop Relat Res. 2006;(442):115-20.

13. Smith WR, Ziran BH, Anglen JO, Stahel PF. Locking plates: tips and tricks. J Bone Joint Surg Am. 2007;89(10):2298-307.

14. Siffri PC, Peindl RD, Coley ER, Norton J, Connor PM, Kellam JF. Biomechanical analysis of blade plate versus locking plate 
fixation for a proximal humerus fracture: comparison using cadaveric and synthetic humeri. J Orthop Trauma. 2006;20(8): 547-54.

15. Ricci WM. Techniques in orthopaedics. Locked plating. Biomech Biol Clin Indic. 2007;22(4 Pt 2):192-6.

16. Lill H, Hepp P, Korner J, Kassi JP, Verheyden AP, Josten C, Duda GN. Proximal humeral fractures: how stiff should an implant be? A comparative mechanical study with new implants in human specimens. Arch Orthop Trauma Surg. 2003;123(2-3): 74-81.

17. Self J, Viegas SF, Buford WL Jr, Patterson RM. A comparison of double-plate fixation methods for complex distal humerus fractures. J Shoulder Elbow Surg. 1995;4(1 Pt 1):10-6.
18. Kristiansen B, Christensen SW. Plate fixation of proximal humeral fractures. Acta Orthop Scand. 1986;57(4):320-3.

19. Bigliani LU, Graig EV, Butters KP. Fraturas do ombro. In: Rockwood Jr CA, Green DP, Bucholz RW. Fraturas em adultos. 3a ed. São Paulo: Manole; 1994. v. 2, p. 855-910.

20. Egol KA, Ong CC, Walsh M, Jazrawi LM, Tejwani NC, Zuckerman JD. Early complications in proximal humerus fractures (OTA Types 11) treated with locked plates. J Orthop Trauma. 2008;22(3):159-64.

21. Checchia SL, Doneux PS, Miyazaki AN, Fregoneze M, Silva LA, Lobo AC, et al. Avaliação do tratamento cirúrgico da fratura em duas partes do colo cirúrgico do úmero com placa PFS 80. Rev Bras Ortop. 2004;39(10):555-67. 